\title{
Correlation of miR-494 expression with tumor progression and patient survival in pancreatic cancer
}

\author{
Y.B. $\mathrm{Ma}^{1,2}$, G.X. Li ${ }^{3}$, J.X. Hü ${ }^{4}$ X. Liu ${ }^{5}$ and B.M. Shi ${ }^{1,6}$ \\ 1'Department of Hepatobiliary Surgery, Shandong Provincial Hospital, \\ ShanDong, China \\ 'Department of Hepatobiliary Surgery, Weifang People's Hospital, Shandong, China \\ 'Department of Gastroenterology, Shouguang People's Hospital, Shandong, China \\ ${ }^{4}$ Department of General Surgery, Shouguang People's Hospital, Shandong, China \\ ${ }^{5}$ Department of Medical Oncology, Affiliated Hospital of Weifang Medical College, \\ Shandong, China \\ ${ }^{6}$ Department of General Surgery, Tongji Hospital of Tongji University, \\ Shanghai, China \\ Corresponding author: B.M. Shi \\ E-mail: tongji_shibaomin@126.com
}

Genet. Mol. Res. 14 (4): 18153-18159 (2015)

Received August 3, 2015

Accepted October 26, 2015

Published December 23, 2015

DOI http://dx.doi.org/10.4238/2015.December.23.2

ABSTRACT. MicroRNA-494 (miR-494) expression is aberrant in various
types of human cancer. However, the prognostic value of miR- 494 in
pancreatic cancer remains unclear. The level of miR- 494 expression
was determined in 99 pairs of primary pancreatic cancer and their
corresponding, adjacent non-tumor tissues by using quantitative
reverse transcriptase polymerase chain reaction. We also analyzed
the associations between miR-494 expression and clinicopathological
features. The survival correlations were analyzed by using the Kaplan-
Meier method and Cox proportional hazards model. The level of miR-
494 expression was significantly downregulated in pancreatic cancer
tissues (mean relative expression level $\pm \mathrm{SD}, 0.48 \pm 0.11)$ as compared
to matched adjacent normal tissues $(1.80 \pm 0.28, \mathrm{P}<0.05)$. We found 
significant correlations between the miR-494 expression levels and TNM stage $(P=0.009)$, lymphatic invasion $(P=0.036)$, vascular invasion $(P$ $=0.011)$, distant metastasis $(P=0.007)$, and tumor grade $(P=0.031)$. Pancreatic cancer patients with a low miR-494 expression level had a shorter overall survival than those with a high miR-494 expression level $(P<0.05)$. Reduced miR-494 expression in pancreatic cancer tissues is correlated with tumor progression and might be an independent, poor prognostic factor for patients with pancreatic cancer.

Key words: microRNA; miR-494; Survival; Prognosis; Pancreatic cancer

\section{INTRODUCTION}

Pancreatic cancer is the sixth leading cause of death due to malignant disease in China and the fourth leading cause of cancer-related death in the USA (Guo et al., 2005; Siegel et al., 2014). Pancreatic carcinogenesis is a multistep process involving multiple genetic and epigenetic alterations (Macgregor-Das et al., 2013). Hence, a better understanding of the molecular mechanisms involved in pancreatic carcinogenesis will be helpful for identification of new and effective prognostic biomarkers.

MicroRNAs (miRNAs) are small non-coding regulatory RNAs that suppress gene expression through partial complementary elements in the 3'-untranslated regions of their target messenger RNAs (mRNAs)(Bartel 2004). miRNAs have been shown to be involved in various critically important physiological processes such as cell proliferation, cell division, cell differentiation, cell apoptosis, tumorigenesis, hematopoiesis, and the nervous system patterning (Ambros, 2004; Harfe, 2005). A particular type of miRNA-miR-494-is downregulated in different types of cancer tissues. Moreover, it is a tumor suppressor and induces cell cycle arrest, cell senescence, and apoptosis, but suppresses cell proliferation (Diakos et al., 2010; Ohdaira et al., 2012; Yamanaka et al., 2012). Li et al. found that downregulation of miR-494 via loss of SMAD4 increased FOXM1 and $\beta$-catenin signaling in pancreatic cancer cells. However, the association between the expression of miR-494 and the clinicopathological characteristics of pancreatic cancer remain unclear. Thus, the aim of this study was to evaluate the clinical significance of miR-494 in pancreatic cancer.

\section{MATERIAL AND METHODS}

\section{Patients and tumor tissues}

The study protocol was approved by the Ethics Committee of Shandong Provincial Hospital affiliated to ShanDong University, and all patients provided written informed consent for the use of their tissues. A total of 99 pairs of human pancreatic cancer tissues and matched normal adjacent pancreatic tissues were obtained during surgery between July 2006 and August 2012 at the Department of Hepatobiliary Surgery, Shandong Provincial Hospital, which is affiliated to ShanDong University. The diagnosis was based on pathological evidence. The specimens were immediately snap-frozen and stored at $-80^{\circ} \mathrm{C}$ for future miR-494 extraction. None of the patients received chemotherapy or radiotherapy before the surgical excision. 


\section{Quantitative reverse transcriptase polymerase chain reaction}

Total RNA was extracted from pancreatic cancer tissues and matched normal adjacent tissues by homogenizing the tissue in Trizol (Invitrogen, USA) according to the manufacturer's instructions. Primers for miR-494 and endogenous control U6 snRNA were obtained from Applied Biosystems (Foster City, USA). The concentration and purity of RNA were determined spectrophotometrically using the NanoDrop ND-1000 (NanoDrop Technologies, USA). cDNA was generated using the PrimeScript RT reagent kit (Takara Co. Ltd., Dalian, China) in $20 \mu \mathrm{L}$ of final reaction volume containing $0.5 \mu \mathrm{g}$ of RNA, $0.5 \mu \mathrm{L}$ Prime-Script RT enzyme mix, $4 \mu \mathrm{L} 5 \mathrm{X}$ PrimeScript buffer, and $1 \mu \mathrm{L} \mathrm{RT} \mathrm{primer.} \mathrm{The} \mathrm{reaction} \mathrm{mixture} \mathrm{was} \mathrm{incubated} \mathrm{at} 42^{\circ} \mathrm{C}$ for $60 \mathrm{~min}$ and at $85^{\circ} \mathrm{C}$ for $5 \mathrm{~min}$. Quantitative real-time polymerase chain reaction was performed to evaluate the miR-494 expression using SYBR Premix Ex Taq (Takara Co. Ltd.) and measured in a LightCycler 480 System (Roche, Basel, Switzerland). The amplification was conducted in the following steps: denaturation at $95^{\circ} \mathrm{C}$ for 10 min followed by 45 cycles of denaturation at $95^{\circ} \mathrm{C}$ for $15 \mathrm{~s}$, annealing at $60^{\circ} \mathrm{C}$ for $30 \mathrm{~s}$, and extension at $72^{\circ} \mathrm{C}$ for $1 \mathrm{~min}$. Relative quantification of miRNA expression was performed using the $2^{-\Delta \Delta C}$ Talue. The raw data were presented as the relative quantity of target miRNA, normalized with respect to U6 snRNA and relative to a calibrator sample.

\section{Statistical analysis}

Statistical analyses were performed using SPSS for Windows version 17.0 (SPSS Inc., Chicago, IL, USA). Differences between the two groups were estimated using the Student $t$-test and the Chi-square test. Overall survival (OS) was measured up to the date of death from any cause or, for living patients, the date of last contact. OS was estimated using the Kaplan-Meier method, and the differences in survival were compared using the log-rank test. A Cox proportional hazards model was used for multivariate analysis. Values of $P<0.05$ were considered statistically significant.

\section{RESULTS}

\section{Expression of miR-494 in pancreatic cancer}

The level of miR-494 expression was significantly downregulated in pancreatic cancer tissues (mean relative expression level $\pm \mathrm{SD}, 0.48 \pm 0.11$ ) when compared with matched adjacent normal tissues $(1.80 \pm 0.28, P<0.05$, Figure 1$)$. The relative miR-494 expression level was classified as high or low in relation to the median value.

\section{Association between miR-494 expression and clinicopathological variables of pancreatic cancer patients}

The relationships between miR-494 expression levels and clinicopathological characteristics in individuals with pancreatic cancer are summarized in Table 1. We found significant correlations between miR-494 expression levels and the TNM stage $(P=0.009)$, lymphatic invasion $(P=0.036)$, vascular invasion $(P=0.011)$, distant metastasis $(P=0.007)$, and tumor grade $(P=$ $0.031)$. However, we did not find a significant association of the miR-494 expression levels with sex $(P=0.62)$, age $(P=0.21)$, tumor size $(P=0.11)$, and tumor location $(P=0.45)$. 


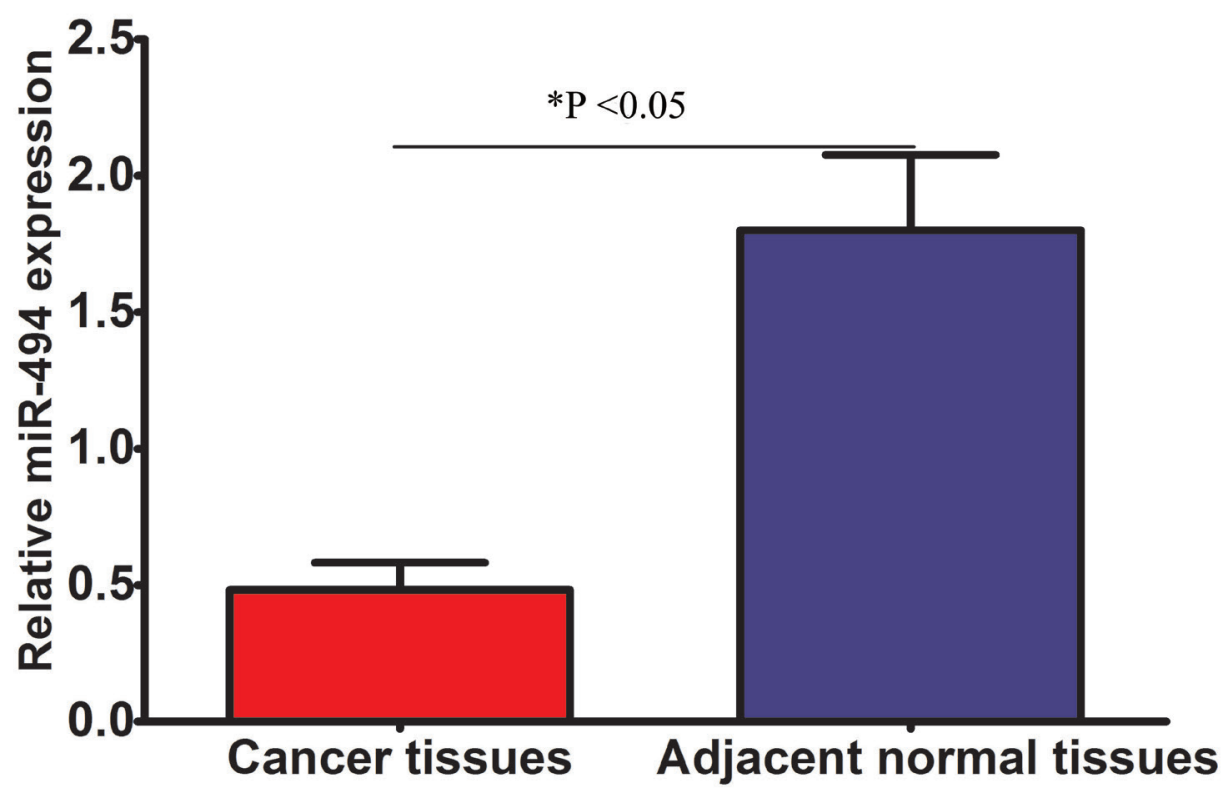

Figure 1. The level of miR-494 expression in pancreatic cancer tissues as compared to matched adjacent normal tissues $(P<0.05)$.

Table 1. Relationship between miR-494 and clinicopathological characteristics in 99 patients with pancreatic cancer.

\begin{tabular}{|c|c|c|c|c|}
\hline \multirow[t]{2}{*}{ Parameters } & \multirow[t]{2}{*}{ Number of cases } & \multicolumn{2}{|c|}{ miR-494 expression } & \multirow[t]{2}{*}{$P$ value } \\
\hline & & Low & High & \\
\hline \multicolumn{5}{|l|}{ Gender } \\
\hline Male & 51 & 27 & 24 & \multirow[t]{2}{*}{0.62} \\
\hline Female & 48 & 17 & 31 & \\
\hline \multicolumn{5}{|l|}{ Age } \\
\hline$<60$ years & 49 & 16 & 33 & \multirow[t]{2}{*}{0.21} \\
\hline$\geq 60$ years & 50 & 28 & 22 & \\
\hline \multicolumn{5}{|l|}{ Tumor Size } \\
\hline$<2 \mathrm{~cm}$ & 67 & 25 & 42 & \multirow[t]{2}{*}{0.11} \\
\hline$\geq 2 \mathrm{~cm}$ & 32 & 19 & 13 & \\
\hline \multicolumn{5}{|l|}{ Location } \\
\hline Head & 58 & 28 & 30 & \multirow[t]{2}{*}{0.45} \\
\hline Other & 41 & 16 & 25 & \\
\hline \multicolumn{5}{|c|}{ Lymphatic invasion } \\
\hline Positive & 48 & 31 & 17 & \multirow[t]{2}{*}{0.036} \\
\hline Negative & 51 & 13 & 38 & \\
\hline \multicolumn{5}{|l|}{ Vascular invasion } \\
\hline Positive & 46 & 30 & 16 & \multirow[t]{2}{*}{0.011} \\
\hline Negative & 53 & 14 & 39 & \\
\hline \multicolumn{5}{|l|}{ Distant metastasis } \\
\hline Positive & 42 & 32 & 10 & \multirow[t]{2}{*}{0.007} \\
\hline Negative & 57 & 12 & 45 & \\
\hline \multicolumn{5}{|l|}{ TNM stage } \\
\hline I/II & 54 & 37 & 17 & \multirow[t]{2}{*}{0.009} \\
\hline III/IV & 45 & 7 & 38 & \\
\hline \multicolumn{5}{|l|}{ Histologic grade } \\
\hline High/moderate & 56 & 33 & 23 & \multirow[t]{2}{*}{0.031} \\
\hline Poor & 43 & 11 & 32 & \\
\hline
\end{tabular}




\section{Association between miR-494 expression and prognosis of pancreatic cancer patients}

To evaluate the prognostic value of miR-494 expression in pancreatic cancer, survival curves were constructed by using the Kaplan-Meier method and compared by using the log-rank test. Pancreatic cancer patients with low miR-494 expression level had a shorter overall survival than those with high miR-494 expression level (Figure 2). The 5-year overall survival rate in the low-expression groups was $12.0 \%$ compared with $49.2 \%$ in the high-expression group (log-rank test, $P=0.036$ ).

To determine whether the expression of miR-494 was an independent risk factor for poor prognosis, both clinicopathological factors and the level of miR-494 expression were evaluated by performing multivariate Cox regression analysis. We found that the miR-494 expression level (hazard ratio $[\mathrm{HR}]=2.85,95 \%$ confidence interval $[\mathrm{Cl}]$ : 1.54-8.45, $\mathrm{P}=0.019$ ) was an independent factor for predicting the overall survival of pancreatic cancer patients (Table 2).

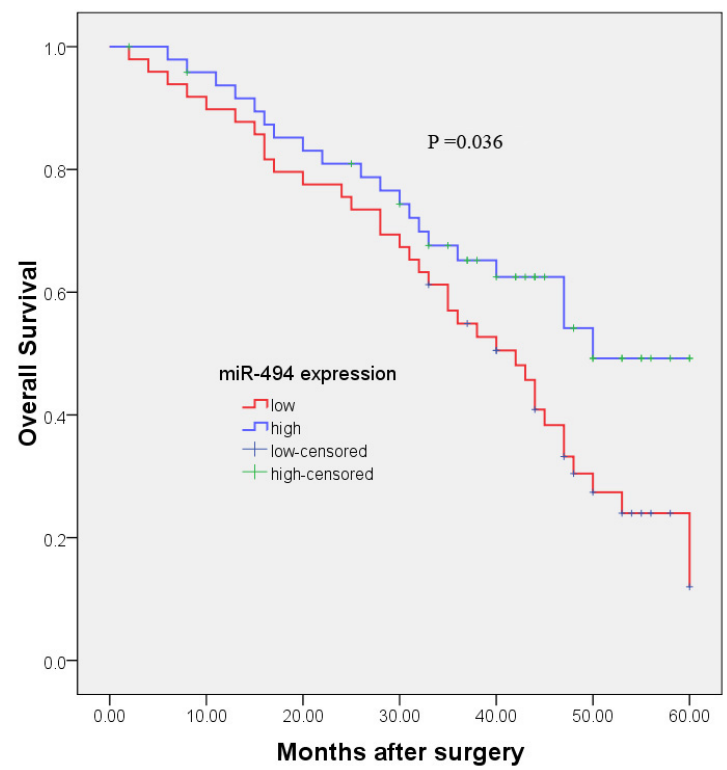

Figure 2. Comparison between the overall survival in pancreatic cancer patients with high and low miR-494 expression levels $(P=0.036)$.

Table 2. Multivariate analysis of factors associated with overall survival in pancreatic cancer patients.

\begin{tabular}{lccc}
\hline Variable & Hazard ratio & $95 \% \mathrm{Cl}$ & $\mathrm{P}$ value \\
\hline Gender & 0.87 & $0.35-1.68$ & 0.45 \\
Age & 1.28 & $0.65-1.54$ & 0.33 \\
Tumor Size & 1.23 & $0.75-2.92$ & 0.24 \\
Location & 1.48 & $0.54-2.62$ & 0.74 \\
Lymphatic invasion & 2.92 & $1.37-10.39$ & 0.016 \\
Vascular invasion & 2.18 & $1.03-16.86$ & 0.008 \\
Distant metastasis & 3.03 & $2.15-18.92$ & 0.012 \\
TNM stage & 2.98 & $1.88-14.12$ & 0.007 \\
Histologic grade & 1.21 & $0.78-8.27$ & 0.06 \\
miR-494 expression & 2.85 & $1.54-8.45$ & 0.019 \\
\hline
\end{tabular}




\section{DISCUSSION}

Although pancreatic cancer has been considerably studied over the past several decades, new and effective treatment regimens are lacking, and chemotherapy and radiation therapy remain ineffective (Neoptolemos et al., 2004). Therefore, surgery is the only curative treatment option; however, only 15-20\% pancreatic cancer patients are eligible for surgery at the time of presentation. Further, of those who undergo successful surgical resection, the 5-year survival rate is only $15-23 \%$ (Simianu et al., 2010). To develop novel treatment strategies for treatment of pancreatic cancer patients, a better understanding of the molecular pathogenesis of pancreatic cancer is required. In addition, accurate prediction of the prognosis for individual pancreatic cancer patients is of great importance, and molecular biomarkers that could serve as prognostic factors would be useful in determining an individualized treatment plan for a pancreatic cancer patient (Hurwitz et al., 1992). However, the biomarkers used in these group of patients today are not satisfactory (Winter et al., 2013), and additional markers need to be tested to fine-tune this process.

Recent studies have indicated that mutations or misexpression in miRNAs are correlated with various human cancers and can act as oncogenes or tumor-suppressor genes by controlling the expression of protein-coding miRNAs (Esquela-Kerscher et al., 2006). Varied expression of miRNAs in normal tissues compared with cancerous tissues and the significant correlation between specific expression and prognosis imply that miRNAs are determinants of certain clinical outcomes (Lu et al., 2005; Volinia et al., 2006). miR-494 has been found to be downregulated in different types of cancer tissues and is a tumor suppressor, as it induced cell cycle arrest, cell senescence, and apoptosis and suppresses cell proliferation (Diakos et al. 2010; Ohdaira et al. 2012; Yamanaka et al. 2012). Ohdaira et al. found that miR-494 suppressed cell proliferation and induced senescence in A549 lung cancer cells (Ohdaira et al. 2012). Furthermore, Romano et al. (2012) found that miR-494 was regulated by ERK1/2 and modulated by tumor necrosis factorrelated apoptosis-inducing ligand-induced apoptosis in non-small-cell lung cancer through BIM downregulation. The findings by Kim et al. (2011) indicated that miR-494 was a negative regulator of KIT in gastrointestinal stromal tumors (GISTs), and overexpressing miR-494 in GISTs might be a promising approach to GIST treatment. Previously, Li et al. found that downregulation of miR-494 via the loss of SMAD4 increased FOXM1 and $\beta$-catenin signaling in pancreatic cancer cells. However, the association between miR-494 expression and the clinicopathological characters of pancreatic cancer remain unclear. In the present study, we found that the level of miR-494 expression was significantly downregulated in pancreatic cancer tissues, and that significant correlations existed between miR-494 expression levels and TNM stage, lymphatic invasion, vascular invasion, distant metastasis, and tumor grade. These results indicated that the miR-494 deregulation was involved in invasion/metastasis of pancreatic cancer. Therefore, the level of miR-494 expression might be correlated with a poor prognosis of patients with pancreatic cancer. To evaluate the prognostic value of miR-494 expression in pancreatic cancer, survival curves were constructed using the Kaplan-Meier method and compared using the log-rank test. Using these analyses, we found that pancreatic cancer patients with low miR-494 expression levels had shorter overall survival than those with high miR-494 expression levels. To determine whether miR-494 is an independent risk factor for poor prognosis, both clinicopathological factors and the level of miR-494 expression were evaluated by performing multivariate Cox regression analysis. Results showed that miR-494 expression level $(\mathrm{HR}=2.85,95 \% \mathrm{Cl}: 1.54-8.45, \mathrm{P}=0.019)$ was an independent factor in predicting the overall survival of pancreatic cancer patients. To the best of our knowledge, this is the first study investigating the clinicopathological and prognostic values of miR-494 in pancreatic cancer. 
In conclusion, reduced miR-494 expression level in pancreatic cancer tissues was correlated with tumor progression and might be an independent poor prognostic factor for patients with pancreatic cancer. In the future, a larger sample population is needed to confirm the prognostic value of miR-494 expression in pancreatic cancer.

\section{REFERENCES}

Ambros V (2004). The functions of animal microRNAs. Nature. 431: 350-355.

Bartel DP (2004). MicroRNAs: genomics, biogenesis, mechanism, and function. Cell. 116: 281-297.

Diakos C, Zhong S, Xiao Y, Vasconcelos GM, et al. (2010). TEL-AML1 regulation of survivin and apoptosis via miRNA-494 and miRNA-320a. Blood. 116: 4885-4893.

Esquela-Kerscher A and Slack FJ (2006). Oncomirs - microRNAs with a role in cancer. Nat. Rev. Cancer. 6: $259-269$.

Guo X and Cui Z (2005). Current diagnosis and treatment of pancreatic cancer in China. Pancreas. 31: 13-22.

Harfe BD (2005). MicroRNAs in vertebrate development. Curr. Opin. Genet. Dev. 15: 410-415.

Hurwitz M, Sawicki M, Samara G and Passaro E Jr. (1992). Diagnostic and prognostic molecular markers in cancer. Am. J. Surg. 164: 299-306.

Kim WK, Park M, Kim YK, Tae YK, et al. (2011). MicroRNA-494 downregulates KIT and inhibits gastrointestinal stromal tumor cell proliferation. Clin. Cancer Res. 17: 7584-7594.

Lu J, Getz G, Miska EA, Alvarez-Saavedra E, et al. (2005). MicroRNA expression profiles classify human cancers. Nature. 435: 834-838.

Macgregor-Das AM and lacobuzio-Donahue CA (2013). Molecular pathways in pancreatic carcinogenesis. J. Surg. Oncol. 107: 8-14.

Neoptolemos JP, Stocken DD, Friess H, Bassi C, et al. (2004). A randomized trial of chemoradiotherapy and chemotherapy after resection of pancreatic cancer. N. Engl. J. Med. 350: 1200-1210.

Ohdaira H, Sekiguchi M, Miyata K and Yoshida K (2012). MicroRNA-494 suppresses cell proliferation and induces senescence in A549 lung cancer cells. Cell Prolif. 45: 32-38.

Romano G, Acunzo M, Garofalo M, Di Leva G, et al. (2012). MiR-494 is regulated by ERK1/2 and modulates TRAIL-induced apoptosis in non-small-cell lung cancer through BIM down-regulation. Proc. Natl. Acad. Sci. U. S. A. 109: 16570-16575.

Siegel R, Ma J, Zou Z and Jemal A (2014). Cancer statistics, 2014. C.A. Cancer J. Clin. 64: 9-29.

Simianu VV, Zyromski NJ, Nakeeb A and Lillemoe KD (2010). Pancreatic cancer: progress made. Acta. Oncol. 49: $407-417$.

Volinia S, Calin GA, Liu CG, Ambs S, et al. (2006). A microRNA expression signature of human solid tumors defines cancer gene targets. Proc. Natl. Acad. Sci. U. S. A. 103: 2257-2261.

Winter JM, Yeo CJ and Brody JR (2013). Diagnostic, prognostic, and predictive biomarkers in pancreatic cancer. J. Surg Oncol. 107: 15-22.

Yamanaka S, Campbell NR, An F, Kuo SC, et al. (2012). Coordinated effects of microRNA-494 induce G(2)/M arrest in human cholangiocarcinoma. Cell Cycle. 11: 2729-2738. 